\title{
Journal-club pour le praticien Journal-Klub für den Praktiker
}

\author{
Une sélection du journal-club pour le praticien \\ de la Clinique de Genève/J.-H. Saurat

\section{Eine Auswahl des Journal-Klubs für den Praktiker der Genfer Klinik/J.-H. Saurat}

\section{Stress et peau: enfin plus qu'une probabilité}

[Arch Dermatol 2001;137:53]

Personne n'oserait aujourd'hui contester le rôle probable du stress dans l'évolution de maladies de la peau. Pourtant les preuves d'un effet du stress sur des fonctions cutanées manquent. La première étude montrant un tel lien est l'œuvre du groupe de Peter Elias de SanFrancisco. Elle montre que la reconstitution de la barrière cutanée après stripping est retardée lors du stress. Les sujets étaient des étudiants en médecine et pharmacie testés avant les examens ou après les vacances, et le stress était mesuré par deux échelles psychologiques validées. Le retard à la reconstitution de la barrière est considéré comme une anomalie significative de la fonction cutanée, par laquelle le stress pourrait influencer l'évolution de dermatoses comme le psoriasis ou la dermatite atopique. Quant aux mécanismes moléculaires liant le stress et cette anomalie épidermique ... à suivre.

\section{Stress und Haut: Endlich mehr als nur eine Vermutung}

[Arch Dermatol 2001;137:53]

Niemand möchte heute wohl im Ernst bestreiten, dass Stress wahrscheinlich zur Entstehung von Hautkrankheiten beiträgt. Und dennoch fehlen Beweise für die Auswirkung von Stress auf die Funktionen der Haut. Die erste Studie, in der ein solcher Zusammenhang aufgezeigt wird, kommt von der Arbeitsgruppe um Peter Elias aus San Francisco. Diese Autoren haben nachgewiesen, dass die Barrierefunktion der Haut nach einer Hautabrasion langsamer wiederhergestellt wird, wenn Stress im Spiel ist. Als Probanden nahmen an dieser Studie Medizin- und Pharmaziestudentinnen und -studenten teil, die vor ihren Prüfungen sowie nach den Ferien getestet wurden.

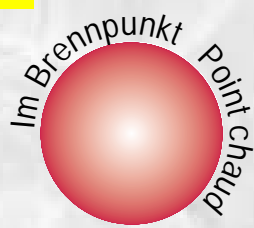

Zur Messung des Stresses wurden zwei validierte psychologische Messskalen verwendet. Der verzögerte Wiederaufbau der Barrierefunktion wird als bedeutende Hautfunktionsstörung eingestuft, die der Entwicklung von Dermatosen wie Psoriasis und atopisches Ekzem unter Stress Vorschub leisten könnte. Die molekularen Zusammenhänge zwischen Stress und dieser epidermalen Störung werden wohl Thema künftiger Beiträge sein.

\section{Les vaisseaux lymphatiques sortent de l'ombre grâce aux VEGF-C et -D}

[J Invest Dermatol Symp Proc 2000;5:14; Nat Med 2001;7:199; Nat Med 2001;7:186; Nat Med 2001;7:192]

Quoi de plus banal en pratique que des mots comme «stase lymphatique», «envahissement lymphatique», etc.... Or le vaisseau lymphatique restait si mal connu que l'explication des mécanismes qui gouvernent ces faits faisait intervenir des concepts qui empruntaient plus à la plomberie qu'à la médecine moléculaire. Cela est du passé, le système lymphatique est redécouvert, avec des promesses d'application thérapeutique. Cela justifie, plus qu'un simple résumé des quatres articles récents, presque une mini-revue: la voici.

\section{La circulation lymphatique}

Les vaisseaux lymphatiques rapportent le fluide extravasé dans la circulation sanguine. Au niveau tissulaire, c'est la pression interstitielle et la contraction musculaire qui assurent le flux lymphatique. Le vaisseau lymphatique s'ouvre plutôt que de se collaber sous l'effet de cette pression, grâce à un système complexe de filaments d'ancrage qui amarrent la paroi du vaisseau au tissu conjonctif environnant. Si ces fibres sont détruites, p.ex. par une inflammation (exemple érysipèle récidivant), les vaisseaux lymphatiques ne s'ouvrent pas et la pression aggrave la stase lymphatique [J Invest Dermatol Symp Proc 2000;5:14]. Il s'agit là de simple microtuyauterie..., mais fragile.

\section{La lymphangiogenèse}

Le système lymphatique doit parfois se régénérer, comme les vaisseaux sanguins (angiogenèse); la médecine moléculaire nous fait découvrir les mécanismes de cette lymphangiogenèse. Le chef d'orchestre est une famille de cytokines appelé VEGF («vascular endothelial growth factor»). On connaît depuis plusieurs années le VEGF et ses récepteurs VEGF-R1 et VEGF-R2, qui assurent l'angiogenèse des vaisseaux sanguins. On a ensuite découvert d'autres VEGF, VEGF-B, -C, -D, -E et d'autres récepteurs VEGF-R3. Puis on a découvert que le VEGF-R3 était muté dans le lymphœdème primaire, ce qui indiquait que VEGF-R3 et les cytokines qui le stimulent, VEGF-C et VEGF-D, jouaient un rôle dans la lymphangiogenèse. Aussitôt on a essayé de reproduire le lymphœdème chez des souris en inhibant la voie de VEGF-R3; ces souris développent en effet un lymphœdème. La bonne nouvelle est que ce lymphœdème est régressif, ce qui indique que l'administration de VEGF-C et VEGF-D pourrait induire une régénération des vaisseaux lymphatiques, notamment chez l'homme. On découvre ainsi que certains lymphœedèmes pourront bénéficier d'un traitement spécifique [Nat Med 2001;7: 199].

\section{La lymphangiogenèse}

\section{et les métastases cancéreuses}

Le développement de vaisseaux lymphatiques dans une tumeur est sans doute un des facteurs de progression et de métastases ganglionnaires. Ce sont aussi les VEGF-C et -D qui interviennent dans ce processus, deux articles le démontrent. En surexprimant soit VEGF-C, soit VEGF-D dans des tumeurs, on induit la formation de nombreux vaisseaux lymphatiques et il en résulte une augmentation des métastases ganglionnaires [Nat Med 2001;7:186; Nat Med 2001;7: 192]. Inutile de souligner que la recherche d'inhibiteurs de VEGF-C et -D ainsi que d'antagonistes du VEGF-R3 va devenir un domaine chaud dans les universités et l'industrie. 


\section{Das Lymphsystem beendet sein Schattendasein dank VEGF-C und -D}

[J Invest Dermatol Symp Proc 2000;5:14; Nat Med 2001;7:199; Nat Med 2001;7:186; Nat Med 2001;7:192]

Wörter wie «Lymphstauung», «Lymphknotenbefall» oder ähnliche erregen im Praxisalltag kaum Aufsehen. Und dennoch wusste man bis vor kurzem über Lymphgefässe so wenig Bescheid, dass man zu ihrer Beschreibung eher Begriffe aus dem Wasserleitungsbau als aus der Molekularmedizin heranzog. Doch damit ist jetzt Schluss - das Lymphsystem ist wiederentdeckt, und therapeutische Anwendungen sind angesagt. Dies soll auch als Rechtfertigung dienen, dass wir anstelle einer kurzen Zusammenfassung der vier kürzlich erschienenen Artikel gleichsam eine «Mini-Rezension» folgen lassen.

\section{Der Lymphkreislauf}

Die Lymphgefässe sammeln die aus dem Blutkreislauf austretende Flüssigkeit. In den Geweben ist der Lymphstrom durch den interstitiellen Druck und die Muskelkontraktionen gewährleistet. Aufgrund eines komplexen Systems von Ankerfäden, welche die Gefässwand an das umgebende Bindegewebe fixieren, haben die Lymphbahnen eher die Tendenz, sich unter der Einwirkung dieses Druckes zu öffnen als zusammenzufalten. Werden diese Fasern durch eine Entzündung zerstört, was beispielsweise bei rezidivierendem Erysipel der Fall ist, öffnen sich die Lymphgefässe nicht mehr, und die Lymphstauung verschlimmert sich durch den Druck [J Invest Dermatol Symp Proc 2000;5:14]. Hier hat man es mit einem - allerdings zerbrechlichen - Mikro-Rohrleitungssystem zu tun.

\section{Die Lymphangiogenese}

Die Lymphbahnen müssen sich von Zeit zu Zeit ebenso erneuern wie die Blutgefässe (Angiogenese). Dank der Molekularmedizin wissen wir über die Mechanismen dieser Lymphangiogenese Bescheid. Den Anstoss dazu vermittelt eine unter der Bezeichnung «vascular endothelial growth factor» (VEGF) zusammengefasste Familie von Zytokinen. Den VEGF und seine Rezeptoren VEGF-R1 und VEGF-R2, welche für die Angiogenese von Blutgefässen verant- wortlich sind, kennt man schon seit mehreren Jahren. In der Folge wurden weitere Wachstumsfaktoren aus dieser Familie - VEGF-B, -C, -D und -E - sowie der Rezeptor VEGF-R3 entdeckt. Daran schloss die Erkenntnis an, dass der VEGF-R3-Rezeptor beim primären Lymphödem mutiert ist. Dies deutet darauf hin, dass der Rezeptor VEGF-R3 und die ihn stimulierenden Zytokine VEGF-C und VEGF-D an der Lymphangiogenese beteiligt sind. Man hat daraufhin versucht, durch Hemmung des VEGF-R3-Signalwegs ein Lymphödem bei der Maus $\mathrm{zu}$ reproduzieren, was tatsächlich auch gelang. Die gute Nachricht ist, dass sich dieses Lymphödem zurückbildet, was vermuten lässt, dass die Verabreichung von VEGF-C und VEGF-D - insbesondere beim Menschen - $z u$ einer Regeneration der Lymphgefässe führen könnte. Dies eröffnet die Möglichkeit, dass sich künftig bestimmte Lymphödeme spezifisch therapieren lassen [Nat Med 2001;7:199].

\section{Lymphangiogenese und}

\section{Krebsmetastasen}

Die Neubildung von Lymphgefässen in Tumoren ist zweifellos einer der Faktoren, die das Fortschreiten von Karzinomen und die Entstehung von Lymphknotenmetastasen begünstigen. Wie aus zwei Artikeln hervorgeht, sind auch hier VEGF-C und -D beteiligt. Eine Überexpression von VEGF-C oder VEGF-D in diesen Tumoren löst die Bildung zahlreicher Lymphgefässe aus, wodurch auch die Lymphknotenmetastasen zunehmen [Nat Med 2001;7:186; Nat Med 2001;7:192]. Man geht wohl richtig in der Annahme, dass die Erforschung von VEGF-C- und -D-Hemmern sowie von VEGF-R3-Antagonisten an Universitäten und in der Pharmaindustrie bald auf Hochtouren laufen wird.

\section{Elastorrhexis: \\ une maladie métabolique dont on pourrait prévenir l'aggravation inéluctable}

[Trends Mol Med 2001;7:13; J Am Acad Dermatol 2001;44:33]

Dans un précédent journal-club, j'avais signalé l'identification du gène muté dans le pseudoxanthome élastique [Nat Genet 2000;25:223, 228] qui code pour une protéine appelée MRP6/ ABCC6, un transporteur impliqué dans la «résistance multiple aux médicaments». Cela signifiait que la fragilité des fibres élastiques qui mène à leur déchirure (elastorrhexis) n'était pas une anomalie de structure de la fibre, mais était due à un mécanisme mystérieux. J. Uitto essaie maintenant d'éclaircir ce mystère, et ses suppositions ouvrent une voie nouvelle permettant d'envisager une prévention des dégâts. La protéine MRP6/ ABCC6 est, pense-t-on, une pompe qui exporte hors de la cellule des peptides anioniques. La panne de cette pompe peut mener à une «intoxication» dont les fibres élastiques font les frais. Il s'agit là d'une situation de maladie métabolique, et certains pensent que des processus d'oxydation pourraient être au centre de ces anomalies. On sait que l'elastorrhexis peut se voir en dehors du pseudoxanthome élastique, notamment dans la $\beta$-thalassémie et le vieillissement photo-induit, où le choc oxydatif joue un rôle. Encore une indication pour les anti-oxydants?

\section{Elastorrhexis: \\ eine Stoffwechselkrankheit, deren bisher unvermeidliche Verschlimmerung verhütet werden könnte}

[Trends Mol Med 2001;7:13; J Am Acad

Dermatol 2001;44:33]

In einer früheren Ausgabe des Journal-Klubs hatte ich auf den Nachweis eines mutierten Gens beim Pseudoxanthoma elasticum hingewiesen [Nat Genet 2000;25:223, 228], das für ein Eiweiss namens MRP6/ABCC6 kodiert, welches als Transportprotein an der Mehrfachresistenz gegen Medikamente beteiligt ist. Das würde demnach bedeuten, dass die Brüchigkeit der elastischen Fasern, die sie zum Reissen (Elastorrhexis) bringt, keine Strukturanomalie der Faser darstellt, sondern auf einem mysteriösen Mechanismus beruht. J. Uitto versucht jetzt, diesem Rätsel auf die Spur zu kommen, und seine Vermutungen eröffnen neue Perspektiven für eine eventuelle Verhütung der Schäden. Man nimmt hierbei an, dass es sich beim Protein MRP6/ABCC6 um eine Pumpe handelt, die anionische Peptide aus der Zelle hinausbefördert. Fällt diese Pumpe aus, würde es zu einer «Vergiftung» kommen, 


\section{Journal-club pour le praticien \\ Journal-Klub für den Praktiker}

der die elastischen Fasern zum Opfer fallen. In diesem Fall hätte man es mit einer Stoffwechselstörung zu tun, und gewisse Autoren vertreten die Meinung, dass diesen Anomalien Oxidationsvorgänge zugrunde liegen. Man weiss, dass die Elastorrhexis unabhängig vom Pseudoxanthoma elasticum auftreten kann, insbesondere bei $\beta$-Thalassämie und lichtbedingter Alterung, bei welcher der oxidative Schock eine Rolle spielt. Könnte dies eine weitere Indikation für Antioxidanzien sein?

\section{Pourquoi le mélanome est-il chimiorésistant et comment le rendre chimiosensible?}

[Nature 2001;409:207]

La chimiothérapie détruit les cellules cancéreuses en activant les voies de leur suicide (apoptose). Les cellules qui évitent ce suicide sont résistantes. Le mélanome, dont nous sommes quotidiennement témoins de la chimiorésistance, était un cas particulier car p53, la protéine qui gouverne l'apoptose, n'est pas mutée dans les cellules du mélanome alors qu'elle est anormale dans la plupart des cancers agressifs chimiorésistants. Dans cet article d'auteurs nord-américains, deux données nouvelles éclaircissent la situation. C'est une protéine en aval de p53 dans la voie de l'apoptose qui est inactive dans le mélanome (elle se nomme Apaf1); mais ce qui fait l'importance pratique de cette observation, c'est que la protéine n'est pas anormale (mutée), elle est seulement inactivée par méthylation et surtout elle peut être réactivée par des inhibiteurs de méthylation. Ainsi des lignées de mélanomes chimiorésistants que l'on traite par ces inhibiteurs de méthylation deviennent chimiosensibles. Nous attendons ces cocktails déméthylants pour nos patients!

\section{Weshalb sind Melanome chemoresistent, und wie kann man sie chemosensibel machen?}

[Nature 2001;409:207]

Eine Chemotherapie zerstört Krebszellen, indem sie die Bahnen für den programmierten Zelltod (Apoptose) ak- tiviert. Zellen, die diesem Zelltod ausweichen, sind resistent. Das Melanom, dessen Chemoresistenz sprichwörtlich ist, stellt einen Sonderfall dar, da das Protein p53, das die Apoptose steuert, in Melanomzellen nicht mutiert ist, während es bei der Mehrzahl der aggressiven chemoresistenten Karzinome in abnormer Form vorliegt. In diesem Artikel nordamerikanischer Autoren gibt es zwei neue Anhaltspunkte, welche die Situation klären. Beim Melanom ist ein Protein (Apaf1) in der Apoptosebahn inaktiv, das unterhalb von p53 liegt. Die praktische Bedeutung dieser Beobachtung liegt nun darin, dass das Protein nicht abnorm (d.h. mutiert), sondern lediglich durch Methylierung inaktiviert ist und - vor allem - dass es durch Hemmstoffe der Methylierung wieder aktiviert werden kann. Auf diese Weise werden chemoresistente Melanomzelllinien, die man mit solchen Hemmern behandelt, chemosensibel. Wir warten auf diese demethylierenden «Cocktails» für unsere Patientinnen und Patienten!

\section{Einfache Antworten zu komplexen Fragen}

\section{Monika Harms}
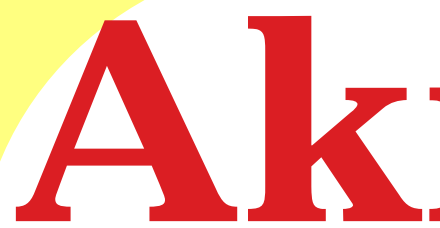

ne

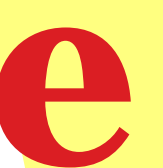

Ein Ratgeber für Patienten

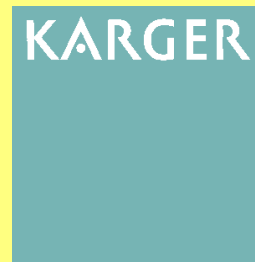

Dieser von der Präsidentin der Schw eizerischen Akne-Gesellschaft, einer anerkannten Expertin, verfasste Ratgeber vermittelt Betroffenen und Angehörigen in leichtverständlicher W eise das Wichtigste über diese komplexe Krankheit.

Im M ittelpunkt des ersten Teils steht die Frage «Was ist Akne?». Die Entstehung der Krankheit, Risiko- und Einflussfaktoren sowie die Diagnose werden detailliert erklärt.

Der zw eite Abschnitt befasst sich intensiv mit den unterschiedlichen Behandlungsmöglichkeiten. Arzneimittel zur innerlichen und äusserlichen Therapie werden vorgestellt, ihre Anwendung und W irkung ausführlich beschrieben. W eitere therapeutische $M$ assnahmen und allgemeine Verhaltensregeln für Akne-Patienten ergänzen diese Erläuterungen.

Am Schluss beantw ortet ein Frage-Antw ort-Katalog die in der Arztpraxis am häufigsten gestellten Fragen zum Thema Akne.

Interessengebiete: Dermatologie; Selbsthilfe

Harms, M. (Genf)

Akne - Ein Ratgeber für Patienten

VIII +80 S., 18 Abb. (farbig), 4 Tab., broschiert, 1997

CHF 20.- / DEM 24.- / USD 17.50

Preisänderungen vorbehalten

DEM - Preise nur für Deutschland, USD-Preise nur für USA

ISBN 3-8055-6457-0 